\title{
Research on Vibration Characteristics of Typical Pipe
}

\author{
Shaoming Yu, Tian Lu, Guo Wei, Yanping Hu \\ Beijing Institute of Structure and Environment Engineering, 100076, Beijing, China
}

\begin{abstract}
Through the study on the vibration characteristics of the typical pipe, it tries to provide the basis for the pipe design and the pipe ground test method. A typical pipe is selected. First, modal analysis is carried out through theoretical analysis and finite element simulation. Then, pipe vibration is studied by finite element simulation and test. The results show that the theoretical results in modal analysis coincide well with those in finite element simulation. The finite element simulation and experimental results are basically consistent in vibration analysis. The reason for the difference is mainly the ideal boundary of simulation. The combination of finite element simulation and test is an important method for the research of pipe reliability and environmental adaptability.
\end{abstract}

\section{Introduction}

The launch vehicle, especially the liquid launch vehicle, is equipped with a large number of pipe systems for the realization of 11ecific functions. As the "blood vessel" of the spacecraft, the reliability and environmental adaptability of the pipe system play an important role in the whole model launch. Because of much failure caused by the pipe's damage, the deep study on the vibration characteristics of the pipe is of great significance to the success of the spacecraft launch. The pipe system is different according to the different use environment. The ground pipe mainly concerns the influence of corrosion and flow induced vibration on the pipe. ${ }^{[1-2]}$ The main part of the spacecraft pipe is the external tension, sound and vibration environment, which affects the pipe. ${ }^{[3-4]}$

In the study of the vibration characteristics of the pipe, there are mainly theoretical analysis, finite element simulation, test verification and other means.

The theoretical analysis of pipe vibration is based on the differential equation of vibration to solve the natural frequency and main vibration mode of pipe system. In the case of low accuracy, the approximate solution is done by Rayleigh-Ritz method and Galerkin method.

Numerical simulation based on finite element method to calculate the pipe system compared to the theoretical analysis more complex, especially relates to damping and forced vibration (random vibration) complex parameters, numerical simulation has great advantages, the simulation results can provide support for the optimization design. ${ }^{[5-}$ 6]

Pipe vibration test plays an important role in the design of spacecraft. It not only verifies the results of theory and simulation, but also plays an irreplaceable role in the assessment of pipe reliability and adaptability.

\section{Typical pipe}

In order to carry on the theory, simulation, experimental research and comparison verification of the vibration characteristics of the pipe, a typical pipe is selected as the research object. As shown in Figure 1, the material of pipe is stainless steel, the inner diameter of the pipe is $32 \mathrm{~mm}$, the wall thickness is $1.5 \mathrm{~mm}$, the length is $800 \mathrm{~mm}$, and the A terminal of pipe is the vibration end (the direction of vibration is $\mathrm{Y}$ ), and the $\mathrm{B}$ end is a fixed end.

The vibration test conditions are shown in Table 1.

Two position paste acceleration sensor in test, respectively in the two three spots line, as shown the position of (1) 、(2) in Figure 1. The positions of the three strain gage are respectively at the two ends and the middle pipe pipe, as shown in Figure 1, 1 8, 10, 12 for the axial strain and 9, 11 for the tangential strain.

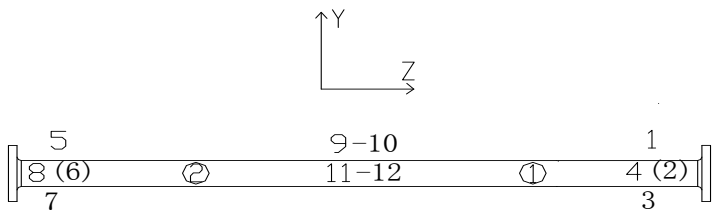

Figure 1 Typical pipe schematic diagram

Table 1. Test conditions for random vibration

\begin{tabular}{ccc}
\hline Frequency range & PSD & RMS \\
\hline $20 \sim 100 \mathrm{~Hz}$ & $3 \mathrm{~dB} / \mathrm{oct}$ & \\
$100 \sim 2000 \mathrm{~Hz}$ & $0.2 \mathrm{~g}^{2} / \mathrm{Hz}$ & $19.74 \mathrm{~g}_{\mathrm{rms}}$ \\
\hline
\end{tabular}




\section{Modal analysis}

The damage of pipe system is mostly caused by the resonance between pipe and structure. When the pipe is designed, the resonant frequency can not be coupled with the resonant frequency of the whole rocket and the whole machine, so the modal information is very important for pipe system.

For the typical pipe studied in this paper, the modal analysis is carried out by means of theoretical research and finite element simulation.

\subsection{Theoretical analysis}

For the pipe in Figure 1, the simplified beam is analyzed. Differential equation for radial vibration of pipe:

$$
E I \frac{\partial^{4} y}{\partial x^{4}}+\rho A \frac{\partial^{2} y}{\partial t^{2}}=p(x, t)-\frac{\partial}{\partial x} m(x, t)
$$

In which, $y(x, t)$ is radial displacement of tube microelement, $p(x, t)$ is the external force acting on the microelement of the pipe, $m(x, t)$ is external torque acting on pipe microelement.

To get the natural frequency and the main mode of the pipe, $p(x, t)=0, m(x, t)=0$, and we can get the general solution of vibration mode of pipe $Y(x)$ :

$$
Y(x)=C_{1} \sin \beta x+C_{2} \cos \beta x+C_{3} \operatorname{sh} \beta x+C_{4} \operatorname{ch} \beta x
$$

$$
\text { While } \beta^{4}=\frac{p^{2} \rho A}{E I}
$$

As shown in Figure 1, the B end of the pipe is fixed, and the A end is vibratory (free), so the boundary condition is:

$$
\left.\begin{array}{l}
\left.Y(x)\right|_{x=0}=0,\left.\frac{d Y(x)}{d x}\right|_{x=0}=0 \\
\left.\frac{d^{2} Y(x)}{d x^{2}}\right|_{x=l}=0,\left.\frac{d^{3} Y(x)}{d x^{3}}\right|_{x=l}=0
\end{array}\right\}
$$

By taking the boundary condition (3) into the general solution equation (2), the frequency equation of a fixed and free equal section straight pipe with one end and one end is obtained:

$$
\cos \beta l \bullet \operatorname{ch} \beta l=-1
$$

The first three roots of the pipe frequency equation are $\beta_{1} l=1.875, \beta_{2} l=4.694 \beta_{3} l=7.855$

Therefore, the natural frequency of the pipe, the main vibration modes are as follows:

$$
\begin{aligned}
& p_{i}=\left(\beta_{i} l\right)^{2} \sqrt{\frac{E I}{\rho A l^{4}}} \\
& Y_{i}(x)=C_{i}\left[\cos \beta_{i} x-\operatorname{ch} \beta_{i} x+r_{i}\left(\sin \beta_{i} x-\operatorname{sh} \beta_{i} x\right)\right]
\end{aligned}
$$

$$
\text { While } r_{i}=\frac{\cos \beta_{i} l+\operatorname{ch} \beta_{i} l}{\sin \beta_{i} l+\operatorname{sh} \beta_{i} l}
$$

The first three natural frequencies of the pipe in Figure 1 are:

$$
\begin{aligned}
& \quad p_{1}=329.8 \mathrm{rad} / \mathrm{s}, \quad p_{2}=2066.8 \mathrm{rad} / \mathrm{s}, \\
& p_{3}=5787.6 \mathrm{rad} / \mathrm{s} \\
& \text { That is } f_{1}=52.5 \mathrm{~Hz}, f_{2}=329.1 \mathrm{~Hz}, \\
& f_{3}=921.6 \mathrm{~Hz},
\end{aligned}
$$

For the pipe in Figure 1, whose B end is fixed and A end undergo the radial vibration, the natural frequency and the main vibration can be obtained results from the theory. Related to damping and forced vibration (random vibration), It is difficult to get the analytical solution in theory and can only be simulated by the finite element method.

\subsection{Finite element simulation}

First, the finite element method is used for modal analysis, which is compared with the theory results. In order to correspond with the theoretical calculation, the finite element method does not consider the flange in modal analysis. The finite element simulation uses the commercial software ABAQUS.

The first three modes (in plane) of the finite element simulation of modal analysis are respectively $53 \mathrm{~Hz}$, $327 \mathrm{~Hz}$ and $897 \mathrm{~Hz}$. The simulation results are shown in Figure 2.

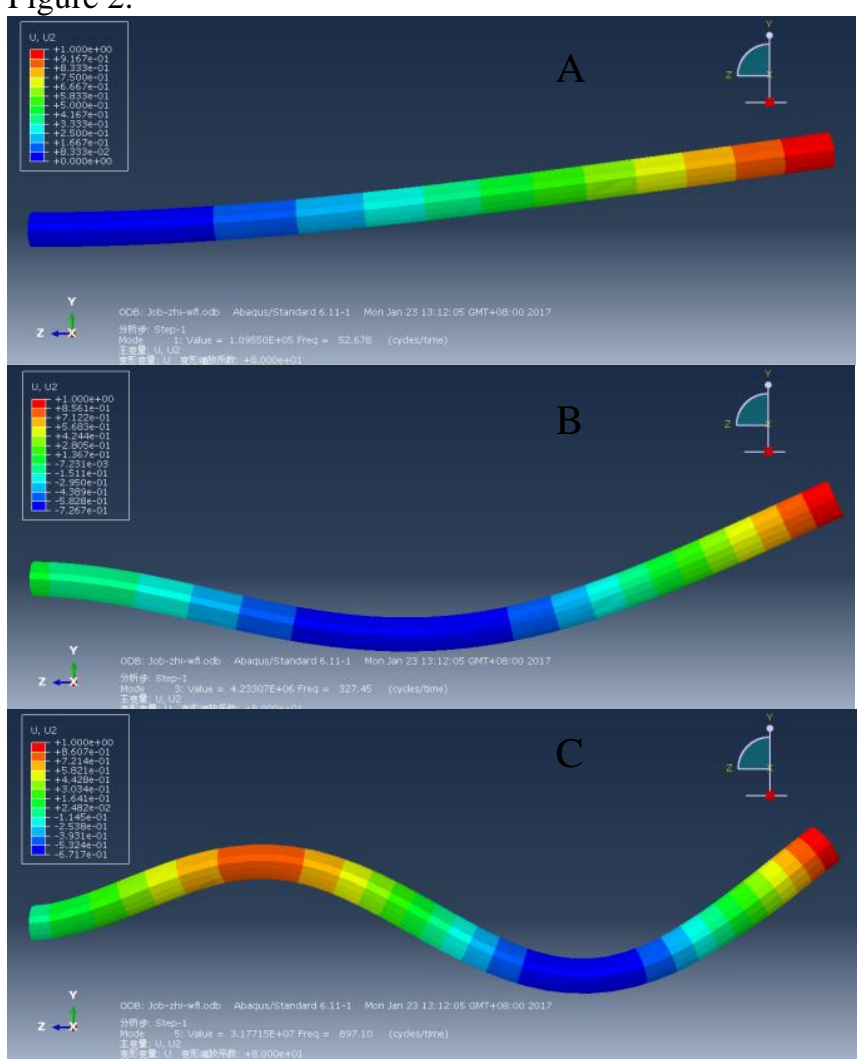

Figure 2 Finite element simulation result diagram of modal analysis 
A) First order mode B) Second order mode C) Third order mode

The finite element results of the pipe modal analysis are in good agreement with the theory. The first order frequency deviation is $0.38 \%$, the second order frequency deviation is $0.49 \%$, and the third order frequency deviation is $2.66 \%$. It can be proved from the results that it is advisable to analyze the pipe with beam model theory, and it also proves the accuracy of the finite element modal analysis.

For the finite element method of pipe vibration analysis, ABAQUS adopts the modal superposition method, which is based on the finite element modal analysis results. The accuracy of finite element modal analysis provides the foundation for the accuracy of vibration analysis.

\section{Vibration analysis}

\subsection{Finite element simulation}

The ABAQUS finite element software is used to analyze the vibration of the typical pipe. The vibration analysis is based on the modal superposition method, and the finite element simulation of the random vibration environment is carried out on the basis of the previous modal analysis results.

The boundary condition of simulation is the vibration environment at the $\mathrm{A}$ end (the loading condition is shown in Table 1) and B terminal is fixed. While random vibration simulation in ABAQUS software can't directly apply the fixed boundary, we add a large mass spot at the fixed end, and tie the fixed boundary with the mass particle. The unidirectional loading method is used for the vibration loading, and the direction is $\mathrm{Y}$ direction in Figure 1.

The result of the cloud is as shown in Figure 3.

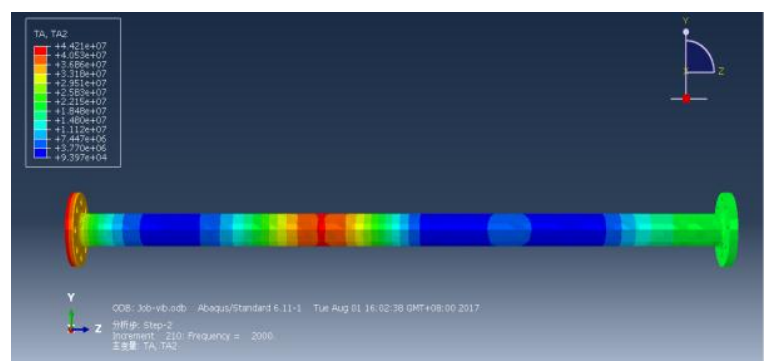

Figure 3 Finite element simulation results of typical pipe vibration

\subsection{Vibration test}

A vibration test of a typical pipe is carried out, and a schematic diagram is shown as shown in Figure 4. B end of the pipe is fixed by the ingot; $A$ end of pipe is exerted vibration; the pipe is connected with the slide by fixture; the loading conditions is shown in Table 1; the loading direction for $\mathrm{Y}$ in Figure 1. For the convenience of comparison, the test is consistent with the finite element simulation boundary in the previous section.

The mounting of the strain gauge and the installation of the acceleration spot are carried out in accordance with figure 1 .

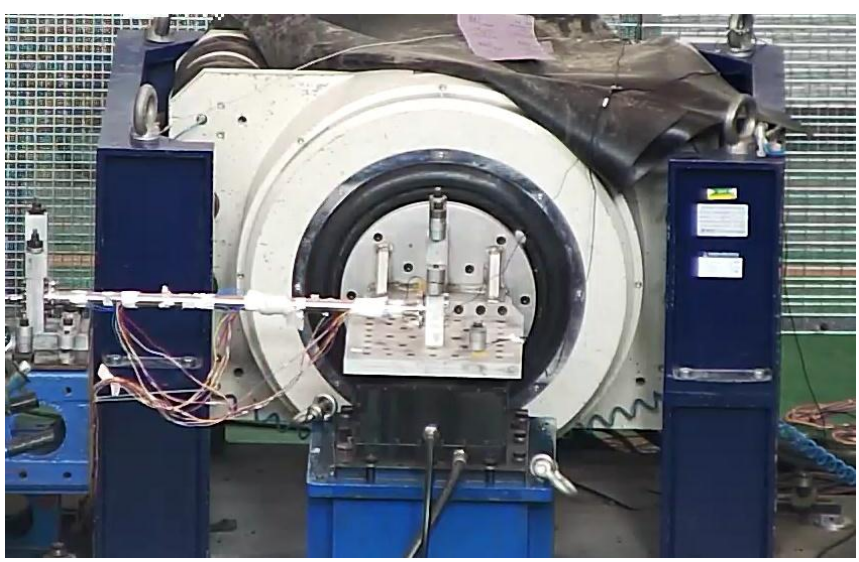

Figure 4 Abridged general view of typical pipe vibration test

\subsection{Comparison between finite element simulation and vibration test}

The results of the finite element simulation are compared with the experimental results.

The root mean square (RMS) comparison of acceleration spots is shown in Table 2; the spot 1's PSD of acceleration comparison is shown in Figure 5; the root mean square strain comparison of each strain measurement spot is shown in Figure 6.

Through comparison, we can get that:

In Table 2, the test results of root mean square acceleration response are larger than that of simulation results. This is because there is a spike in the high frequency section due to interference and other factors in the test process. When the root mean square is calculated, the experimental value is obviously greater than that of the finite element simulation.

In Figure 5, simulation of resonant frequency is high, this is because the simulation boundary is more ideal, which cannot be strictly fixed in test. The finite element simulation has lower peak, this is because the ideal damping and there are no additional mass sensor in finite element simulation. The simulation results are smooth in high frequency, which is due to no circuit interference, no control iteration, no system other structural signal interference (such as screw loosening, shaking table percussion, etc.).

In Figure 6, the root mean square strain ratio data of simulation is smaller than that of the test. The strain of the vibration end is larger than that of the fixed end, and the simulation results are more obvious. The strain on the upper and lower surface of the pipe is larger than that of the neutral layer, and the strain at the neutral layer is small, which is consistent with the bending theory, and the simulation results are consistent. 
Table 2. Acceleration response contrast

\begin{tabular}{ccc}
\hline No. & $\begin{array}{c}\text { RMS in the } \\
\text { test }\left(g_{\text {rsm }}\right)\end{array}$ & $\begin{array}{c}\text { RMS in the } \\
\text { simulation }\left(g_{\text {rsm }}\right)\end{array}$ \\
\hline 1 & 49.656 & 32.224 \\
2 & 43.218 & 31.724 \\
\hline
\end{tabular}

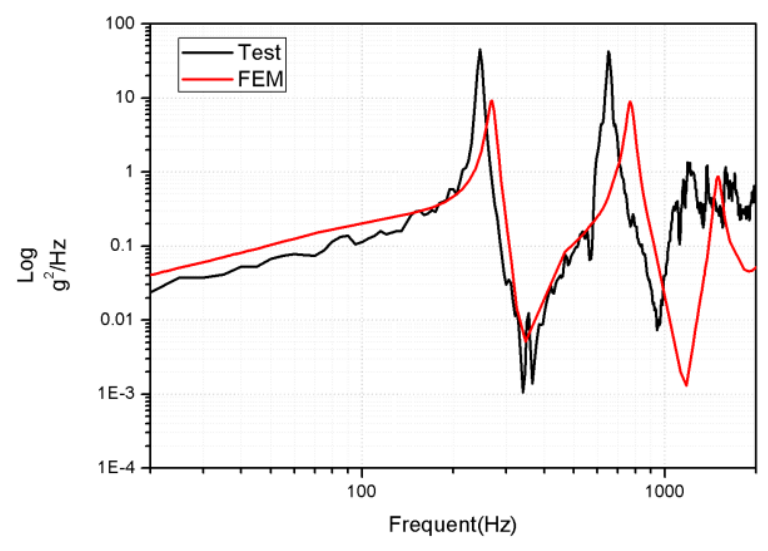

Figure 5 Acceleration PSD contrast of spot 1

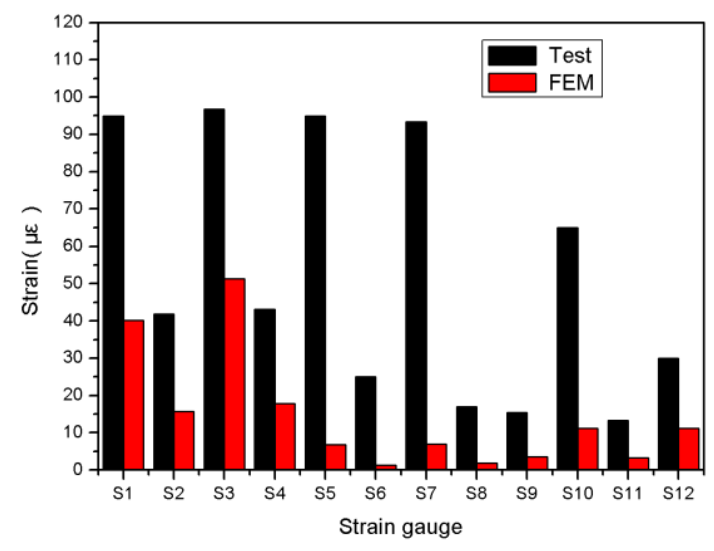

Figure 6 Root mean square strain contrast

\section{Summary}

In the recent model launching process, the pipe abnormality appears many times. The reliability of the pipe and the adaptability of the environment are concerned. The research on the pipe of the spacecraft is of great significance and is facing the urgent need.

Pipe failure is mostly caused by resonance. At present, a number of rockets have begun to carry out frequency management, requiring pipe design frequency to avoid the resonance frequency of whole machine and whole rocket.

Finite element simulation is an important method of pipe research. In actual work, we should strengthen combination of pipe test and finite element simulation, improve efficiency of test through finite element simulation, and verify the accuracy of simulation and repair model through test.

\section{References}

1. M. E. Stevenson, H. C. Iwand, J. L. McDougall, et al. Failure Analysis of an Aluminum Chiller Pipe by Experimental Simulation and Stress Analysis[J]. Journal of Failure Analysis and Prevention. 2017,17(5):1090-1098.

2. Polynikis Vazouras, Spyros A. Karamanos. Structural Behavior of Buried Pipe Bends and Their Effect on Pipe Response in Fault Crossing Areas[J] Bulletin of Earthquake Engineering , 2017,15(11):4999-5024.

3. Yong Bai, Ting Liu, Weidong Ruan, et al. Mechanical Behavior of Metallic Strip Flexible Pipe Subjected to Tension[J] Composite Structures. 2017,170:1-10.

4. P. Dunst, T. Hemsel, W. Sextro. Analysis of Pipe Vibration in An Ultrasonic Powder Transportation System[J] Sensors and Actuators A: Physical. 2017,263:733-736.

5. Wei G, Yu Shaoming, Lu Tian, et al. Environmental Testing Technology of Pipe System[J] Missiles and Space Vehicles, 2017, 3(5):93-96.

6. Yu Shaoming, Lu Tian, Wei G, et al. Research on Vibration of Pipe Based on Strain-analysis[J] Journal of Dynamics and Control, 2017, 15(6):512-517. 